Context The improvement work was done in a neonatal unit inUK. It involved improvement in the care of newborns and included feedback from parents.

Problem The survey was done to assess parent's experiences of neonatal services, identify problems and get an understanding of how the quality of care can be improved. This helped us in developing a culture of continuous improvement that involves and is informed by parents, maintains high quality care for babies and their families and encourages the unit to continually raise the bar in provision of family-centred care.

Study design A questionnaire was sent to parents whose babies were discharged home over 4 months from December 2013 to March 2014. Parents were ineligible for inclusion if the care of the baby was re-oriented, if the baby had been taken into care or discharged to a post-natal ward. The Patient list was identified from Badger net. A Questionnaire and letter of introduction was prepared in consultation with medical and nursing staff. The questionnaire was sent by post once the baby had been discharged. Parents were reminded by phone a week later. Responses were returned to the neonatal secretaries by post.

Assessment of problem and analysis of its causes It was encouraging to see parents reporting positive experiences. There were problems which were identified from the feedback. The problems identified were parents were given inconsistent feeding plans and strategies from nursing staff, majority of parents of babies transferred to or from the unit felt the need to be with their baby during transfer, parents are asked to leave the ward during ward rounds to protect other babies' confidentiality and then usually relied on the nurses for feed back, it was felt by parents that the unit was outdated, overcrowded and stressful "not fit for purpose" and parents coming from outside the city felt the need for a day room and space for families. This will encourage their involvement in the care of their babies.

Strategy for change New NHS trust infant feeding policy and guidelines were formulated. Nursing staff were trained in regard to the policy. There is a need for a full-time breast feeding nurse, If possible, parents will accompany their baby on transfers, an improvement tree was placed in the unit for parents. This is easy to access and will help parents to comment on their experience and offer suggestions, parents will be encouraged to remain with their baby during ward rounds. This will help to involve them in the care of their baby and to update them, parents of babies who are likely to be in the unit for a prolonged time will also be given weekly updates by senior medical staff and plans for a new Women's hospital (2020) are underway and a parent focus group will be involved in the project to ensure their needs are met.

Measurement of improvement The survey will be repeated to see the effect of changes in strategy. Outcomes which have been encouraging have also been monitored by monitoring feeding policy and breastfeeding rates through Badger net and positive feedback from parent comments on the improvement tree.

Lessons learnt and message for others Most parents would like to be present during ward rounds; an opportunity to be updated by medical and nursing staff and allows them to be more involved in the day to day care of their baby; an improvement tree is a good way of getting feedback on a regular basis; Neonatal unit is a stressful environment and parents would benefit from a Day room and where possible, parents should be transported with their babies.

\section{G580(P) CHILD SAFEGUARDING CHECKLIST IN THE EMERGENCY CARE SETTING: IMPROVING CHECKLIST COMPLETION RATES}

S McLoughlin, S Khan. Emergency Care Centre, Royal Albert Edward Infirmary, Wigan, UK

10.1136/archdischild-2015-308599.529

Context This improvement work was done in the Paediatric Emergency Care Centre (PECC) of a busy district general hospital, which sees about 20,000 children every year. The work involved a collaboration of the nursing and medical staff in the PECC.

Problem When a child attends the PECC, a set of notes is generated which includes a Safeguarding Checklist on the second page. It is the responsibility of the clerking doctor to complete this for every child they see. It contains ten questions aimed at identifying children who could have experienced non-accidental injury (NAI). An audit showed that this checklist was only being completed $20 \%$ of the time. This was unacceptable as it put children at risk and contravened a basic trust policy.

Assessment of problem and analysis of its causes To establish why checklist completion was poor, a random sample of nonconsultant emergency doctors was given an anonymous questionnaire. The questionnaire, consisting of 5 multiple choice questions and a final open question, aimed to establish the barriers facing staff completing the checklist. A process map outlining a child's journey through the emergency floor and the above audit made it clear that the checklist was a barrier. Feedback indicated that the checklist itself was busy, unclear and difficult to use. As a result, I set about redesigning the checklist.

Intervention The Safeguarding Checklist now consists of 9 questions with the option to tick "yes", "no" or "not applicable" for each of them. Once they have been completed, there are instructions on how to categorise your findings into "green", "yellow" or "red", indicating the level of risk. Following this, there are bullet point directions of the next steps to take for each category.

Strategy for change The redesigned checklist was integrated in to the new PECC notes, replacing the previous version. The clerking clinicians complete the checklist but nursing staff assist in prompting them to do so. The questionnaire results were presented at a departmental meeting. Once the first draft of the redesigned checklist was completed, it was shown to PECC staff for feedback, which was incorporated in the final version.

Measurement of improvement Following the introduction of the new checklist, the notes of all children under five were audited five days a week to measure how often the checklist was being completed. Results after five months show it is being completed on average $65.6 \%$ of the time. Although this is an increase from $20 \%$, it still does not meet our aim of $100 \%$.

Effects of changes There is clearly an improvement in completion of the checklist but we aim to improve further. By increasing the use of the checklist, we hope to avoid further serious case reviews and promote a safeguarding culture within PECC.

It was difficult to bring together feedback from different sources to produce the final checklist. It was also challenging to create something that was simple and clear but still included all the important information.

Lessons learnt I learnt staff engagement, PDSA cycles and process mapping are key to making improvements. I also learnt that having an open and safe environment to express opinions leads to the some of the best suggestions. 
Next time, I would survey paediatric doctors and ENPs in addition to emergency care doctors as they also complete the checklist while clerking.

Message for others Without regular measurements, we could not tell if the checklist was being used. To find out it is being used poorly through a serious case review is not acceptable. By regular measurement, we have weekly data which provides more detailed information on completion rates. Staff engagement was vital to improving the checklist and increasing compliance, as detailed above.

\section{G581(P) IMPLEMENTATION OF A PAEDIATRIC PRESCRIBING POCKETGUIDE}

PAC Stilwell. Intensive Care, Royal Brompton Hospital, London, UK

\subsection{6/archdischild-2015-308599.530}

Context Neonatology department in a busy district general hospital.

Problem The project set out to address inpatient prescription errors which could result in illegal prescriptions and patient harm.

Assessment of problem and analysis of its causes I collected data from 20 random drug charts and used a checklist to identify the incidence and types of prescribing errors on in-patient drug charts for patients on Special Care Baby Unit and Neonatal Intensive care Unit. After completing the data collection, I met with paediatric pharmacists and departmental doctors and nurses to determine why certain prescribing erros were being made.

Intervention I wrote, designed and developed a pedaitric prescribing pocket guide to clarify local and national guidance regarding paediatric prescribing. I used my own drawings to make a user-friendly guide.

Study design Audit.

Strategy for change I worked with management and senior staff to get the paediatric prescribing guide approved and authorised. It has subsequently been published on the hospital intranet and is available as hardcopies in clinical areas. I used email and departmental meetings to advertise the new prescribing guide, as well as educating new arrivals about paediatric prescribing.

Measurement of improvement To measure the effects of my intervention, I re-audited the incidence and types of prescribing errors on in-patient drug charts. I compared the overall mean prescribing error rate, as well as specific prescribing errors.

Effects of changes Re-audit showed a reduction in mean prescribing error rate, although there is still room for improvement. This has led to clearer prescriptions and fewer uncertainties surrounding delivery of medication. Subjective feedback has reported that the guide has improved communication between multi-disciplinary team members reviewing and using drug charts.

Lessons learnt This project has taught me the need to collect feedback from different team members (pharmacists, nurses, doctors, specialist teams) to identify the reasons behind prescribing errors. I have also learnt how to develop new guidelines and the steps that are needed to go through in order to get new guielines approved and disseminated.

Message for others It is important to involve senior members of staff when developing new guidelines and to be persistent, yet realistic when it comes to implementing new guidelines e.g. printing the guidelines for all new arrivals is expensive whereas making the guidelines available on the trust intranet is cheap and accessible to all healthcare staff.

\section{G582(P) OPERATIVE NOTE QUALITY IMPROVEMENT - EXAMINING COMPLIANCE WITH THE ROYAL COLLEGE OF SURGEONS OF ENGLAND GUIDELINES IN A PAEDIATRIC GENERAL SURGICAL UNIT}

A Ryan, T Reeves, K Kuti, M Flett. Paediatric General Surgery, Royal Hospital for Sick Children Yorkhill, Glasgow, UK

\subsection{6/archdischild-2015-308599.531}

Context Operative notes should be documented "clearly, accurately and legibly" - as endorsed by the GMC's Good Medical Practice. The RCS Eng. Guidelines (2014) advocate contemporaneous, complete surgical records. This audit identified significant shortcomings in operative documentation amongst paediatric general surgical cases in a tertiary care centre. Data collection was performed by two FY1s and a Surgical Registrar.

Problem Surgical operation notes necessitate accurate documentation from both a medico-legal and professional perspective; whilst comprehensive records facilitate post-operative patient management. However; in practice they are frequently substandard, thus raising concerns towards patient safety. Current operative note templates within our unit fail to comply with relevant guidelines.

Assessment of problem and analysis of its causes We identified poorly-documented details within general surgical operation notes using a data collection tool incorporating pertinent, recently-published guidance. Areas for improvement included: patient's weight, type of procedure performed (i.e. emergency vs. elective), whether surgery was with or without complication, and antibiotic prophylaxis. Results attained were compared against data from previous audits of a similar nature (carried out in 2010 and 2011 respectively).

Intervention Our proposed intervention involves promoting typed documentation of operative notes, whilst advocating their transition from paper text to an electronic format. We plan to achieve this through usage of a unique, pre-existing computerised system developed by a consultant within the department, which additionally populates our current Trakcare system upon document creation. We hope to educate the entire general surgical team in its practice.

Furthermore, we will introduce informative posters highlighting the RCS Eng. guidelines and reiterating imperative operative details within the main theatres and day surgery unit. Letters detailing the audit's key findings and our intended strategy for change were sent to all consultants and surgical trainees.

Study design Prospective analysis of 138 general surgical operative notes identified over a 2 -week period. We created a 26 point core checklist incorporating both RCS (Eng.) 2014 and GMC "Good Medical Practice" 2013 guidance. We then audited elective and emergency operative documentation against this standardised proforma. Data collected was subsequently evaluated once compiled onto a computerised spreadsheet (Table 1). Strategy for change Our findings were presented at the weekly departmental meeting, where we emphasised the need for operative note improvement. Proposed interventions were then implemented at the earliest opportunity. In particular, details not previously recorded, yet strongly advocated, by the RCS Eng. were promoted (e.g. anticipated blood loss, DVT prophylaxis etc.). 